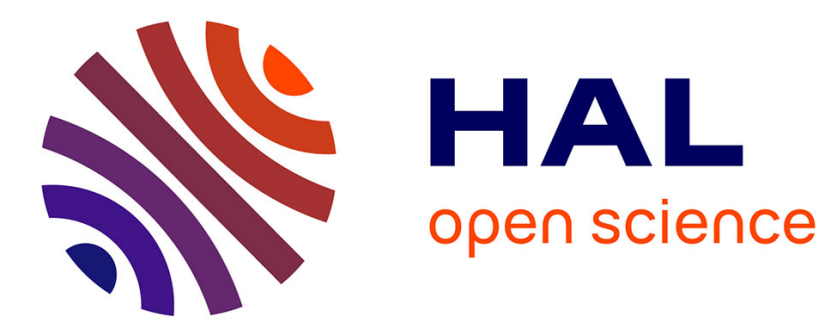

\title{
Convex liftings-based robust control design
}

Ngoc Anh Nguyen, Sorin Olaru, Pedro Rodriguez-Ayerbe, Michal Kvasnica

\section{To cite this version:}

Ngoc Anh Nguyen, Sorin Olaru, Pedro Rodriguez-Ayerbe, Michal Kvasnica. Convex liftings-based robust control design. Automatica, 2017, 77, pp.206- 213. 10.1016/j.automatica.2016.11.031 . hal01509316

\section{HAL Id: hal-01509316 \\ https://hal-centralesupelec.archives-ouvertes.fr/hal-01509316}

Submitted on 5 Mar 2020

HAL is a multi-disciplinary open access archive for the deposit and dissemination of scientific research documents, whether they are published or not. The documents may come from teaching and research institutions in France or abroad, or from public or private research centers.
L'archive ouverte pluridisciplinaire HAL, est destinée au dépôt et à la diffusion de documents scientifiques de niveau recherche, publiés ou non, émanant des établissements d'enseignement et de recherche français ou étrangers, des laboratoires publics ou privés. 


\title{
Convex liftings based robust control design
}

\author{
Ngoc Anh Nguyen ${ }^{a}$, Sorin Olaru ${ }^{a}$, Pedro Rodriguez-Ayerbe ${ }^{a}$, Michal Kvasnica $^{b}$ \\ ${ }^{a}$ Laboratory of Signals and Systems, CentraleSupélec-CNRS-Université Paris Sud, Gif-sur-Yvette, France. \\ ${ }^{\mathrm{b}}$ Department of Information Engineering and Process Control, Slovak University of Technology in Bratislava, Slovakia.
}

\begin{abstract}
This paper presents a new approach for control design of constrained linear systems affected by bounded additive disturbances and polytopic uncertainties. This method hinges on so-called convex liftings which emulate control Lyapunov function by providing a constructive framework for optimization based control implementation. It will be shown that this method can guarantee the recursive feasibility and robust stability in the sense of Lyapunov. Finally, a numerical example will be presented to illustrate this method.
\end{abstract}

Key words: Convex liftings, robust control, bounded additive disturbances, polytopic uncertainties, PWA Lyapunov function.

\section{Introduction}

Originated in Lyapunov (1907), Lyapunov stability becomes a fundamental concept in control theory. In stability analysis, a Lyapunov function is usually of use to prove closed loop stability or robust stability, see Molchanov and Pyatnitskiy (1989); Polanski (1995). On the other hand, in control design, control Lyapunov functions are usually employed to design stabilising/robust controllers, see among others Khalil (2002); Zubov and Boron (1964). Accordingly, whenever such control Lyapunov functions are used in optimization based strategies, these should be chosen such that the recursive feasibility and closed loop stability are all fulfilled. Different classes of control Lyapunov functions have been proposed in control theory. In the context of linear quadratic control, infinite/finite quadratic cost functions usually serve as control Lyapunov functions, as shown in Anderson and Moore (2007); Chmielewski and Manousiouthakis (1996). Particularly, in linear model predictive control, such a control Lyapunov function has been used to design robust controllers to cope with polytopic uncertainties, leading to a linear matrix inequality problem, see Kothare et al. (1996). Polyhedral control Lyapunov functions have also been exploited in several studies e.g. Gutman and Cwikel (1987); Blanchini (1995, 1994); Lazar (2010); Nguyen et al. (2015a), since they lead to simple design procedures; i.e. composed of linear constraints.

Email addresses: Ngocanh. Nguyen@supelec.fr (Ngoc Anh Nguyen), Sorin.Olaruecentralesupelec.fr (Sorin Olaru), pedro.rodriguez@centralesupelec.fr (Pedro Rodriguez-Ayerbe), michal.kvasnica@stuba.sk (Michal Kvasnica).
In the same line with the studies in Gutman and Cwikel (1987); Blanchini (1994); Nguyen (2014); Grammatico et al. (2014), this paper presents an attempt to use convex liftings in the design of robust controllers for discrete-time linear systems affected by bounded additive disturbances and polytopic uncertainties which can serve as control Lyapunov functions. This method is shown to guarantee the recursive feasibility and closed loop stability. In terms of implementation, this only requires resolution of a linear programming problem at each sampling instant.

\section{Notation and Definitions}

Throughout this paper, $\mathbb{N}, \mathbb{N}_{>0}, \mathbb{R}, \mathbb{R}_{+}$denote the set of nonnegative integers, the set of positive integers, the set of real numbers and the set of nonnegative numbers, respectively. For ease of presentation, with a given $N \in \mathbb{N}_{>0}$, by $\mathcal{I}_{N}$, we denote the index set: $\mathcal{I}_{N}=\left\{i \in \mathbb{N}_{>0} \mid i \leq N\right\}$.

A polyhedron is the intersection of finitely many closed halfspaces. A polytope is a bounded polyhedron. If $P$ is an arbitrary polytope, then by $\mathcal{V}(P)$, we denote the set of its vertices. If $\mathcal{S}$ is an arbitrary set, then $\operatorname{conv}(\mathcal{S})$ denotes the convex hull of $\mathcal{S}$. Also, for a full dimensional set $\mathcal{S}$, by $\operatorname{int}(S)$, we denote the interior of $\mathcal{S}$. Further, we use $\operatorname{dim}(\mathcal{S})$ to denote the dimension of its affine hull.

Given a set $\mathcal{S} \subset \mathbb{R}^{d}$ and a matrix $A \in \mathbb{R}^{d \times d}$, then $A \mathcal{S}$ is defined as follows: $A \mathcal{S}=\{A s \mid s \in \mathcal{S}\}$. Also, for any vector $x \in \mathbb{R}^{d}, \rho_{\mathcal{S}}(x)$ is defined as follows: $\rho_{\mathcal{S}}(x)=$ $\min _{y \in \mathcal{S}} \sqrt{(y-x)^{T}(y-x)}$. 
Given two sets $\mathcal{S}_{1}, \mathcal{S}_{2} \subset \mathbb{R}^{d}$, their Minkowski sum is denoted by $\mathcal{S}_{1} \oplus \mathcal{S}_{2}$ and is defined by:

$$
\mathcal{S}_{1} \oplus \mathcal{S}_{2}=\left\{y_{1}+y_{2} \mid y_{1} \in \mathcal{S}_{1}, y_{2} \in \mathcal{S}_{2}\right\}
$$

Also, $\mathcal{S}_{1} \backslash \mathcal{S}_{2}$ is defined as follows:

$$
\mathcal{S}_{1} \backslash \mathcal{S}_{2}:=\left\{x \in \mathbb{R}^{d} \mid x \in \mathcal{S}_{1}, x \notin \mathcal{S}_{2}\right\} .
$$

\section{Problem settings}

In this paper, we consider a discrete-time linear system:

$$
x_{k+1}=A(k) x_{k}+B(k) u_{k}+w_{k},
$$

where $x_{k}, u_{k}, w_{k}$ denote the state, control variables and additive disturbance at time $k$. The state space matrices $[A(k) B(k)]$ are time-varying and assumed to belong to an uncertainty matrix polytope denoted by $\Psi$ and defined below:

$$
[A(k) B(k)] \in \Psi=\operatorname{conv}\left\{\left[A_{1} B_{1}\right], \ldots,\left[A_{L} B_{L}\right]\right\} .
$$

The state, control variables and disturbances are subject to constraints:

$$
x_{k} \in \mathbb{X} \subset \mathbb{R}^{d_{x}}, u_{k} \in \mathbb{U} \subset \mathbb{R}^{d_{u}}, w_{k} \in \mathbb{W} \subset \mathbb{R}^{d_{x}},
$$

where $d_{x}, d_{u} \in \mathbb{N}_{>0}$, and $\mathbb{X}, \mathbb{U}, \mathbb{W}$ are polytopes containing the origin in their interior.

The objective is to find robust control laws which can cope with bounded additive disturbances and polytopic model uncertainties such that the closed loop is robustly stable. It is clear that if $w_{k}$ is unknown, one cannot expect to guarantee asymptotic stability of the origin. In this case, asymptotic stability is replaced with an ultimate boundedness concept Khalil (2002); Kofman et al. (2007) or input to state stability Jiang and Wang (2001).

\section{Robust control design based on convex liftings}

\subsection{Robust positively invariant sets}

Positively invariant sets have been studied over several decades. Due to their relevance in control theory, they turn out to be useful in many control related studies e.g. Bitsoris (1988b,a); Bitsoris and Vassilaki (1995); Blanchini and Miani (2007); Kerrigan (2001). The definition of a robust positively invariant set for system (1) is recalled below.

Definition 4.1 Given an admissible control law $u_{k}=$ $K x_{k} \in \mathbb{U}$, a set $\Omega \subseteq \mathbb{X}$ is called robust positively invariant with respect to (1) if

$$
(A(k)+B(k) K) \Omega \oplus \mathbb{W} \subseteq \Omega, \forall[A(k) B(k)] \in \Psi,
$$

where $\Psi$ is defined in (2).
To compute such a robust positively invariant set $\Omega$, it is important to choose an appropriate unconstrained control law to cope with given bounded additive disturbances and polytopic uncertainties. In case polytopic uncertainties are not taken into account, such a control law $u_{k}=K x_{k}$ can be computed from the Ricatti equation for some positive definite weighting matrices $Q, R$ in the classical linear quadratic control design.

Otherwise, this control law should satisfy that there exists a Lyapunov function $V(x): \mathbb{R}^{d_{x}} \rightarrow \mathbb{R}_{+}$such that

$$
V\left((A(k)+B(k) K) x_{k}\right)-V\left(x_{k}\right)<0, \forall[A(k) B(k)] \in \Psi .
$$

The computation of such a gain $K$ was studied in e.g. Kothare et al. (1996). A simpler formulation is presented below:

$$
\min _{Z, Y}-\log \operatorname{det}(Z)
$$

subject to

$$
\begin{aligned}
& Z=Z^{T}>0 \\
& {\left[\begin{array}{cc}
Z & \left(A_{i} Z+B_{i} Y\right)^{T} \\
A_{i} Z+B_{i} Y & Z
\end{array}\right]>0, \forall i \in \mathcal{I}_{L} .}
\end{aligned}
$$

Then, gain $K$ is determined by

$$
K=Y Z^{-1}
$$

It is already known that the above formulation is a linear matrix inequality (LMI) problem and is solvable by using semidefinite programming. Interested readers can find details in Boyd et al. (1994).

With respect to the state feedback $u_{k}=K x_{k}$, the computation of a robust positively invariant set $\Omega$ for system (1) has been put forward in Nguyen (2014), as a simple extension of the idea presented in Gilbert and Tan (1991). Note also that prominent studies on the computation of the maximal and minimal positively invariant sets for a linear, discretetime invariant system affected by bounded additive disturbances can be found in Kolmanovsky and Gilbert (1998); Rakovic et al. (2005). Still, in case system (1) is not affected by additive disturbances, then the minimal robust positively invariant set coincides with the origin due to its asymptotic stability i.e. $\Omega=\{0\}$.

Without loss of generality, we are interested hereafter in the case $\Omega \subseteq \mathbb{X} \subset \mathbb{R}^{d_{x}}$ represents a full-dimensional set.

\subsection{Domain of attraction}

Given a robust positively invariant set $\Omega$ associated with an admissible state feedback $u=K x \in \mathbb{U}$ for all $x \in \Omega$, the domain of attraction is defined as the set of all points in 
$\mathbb{X}$ which can be driven to $\Omega$, see Khalil (2002). More precisely, the domain of attraction contains all points $x_{0} \in \mathbb{X}$ such that there always exists control law satisfying constraints (3) which is able to steer the state to $\Omega$ as $k \rightarrow \infty$ i.e. $\lim _{k \rightarrow \infty} \rho_{\Omega}\left(x_{k}\right)=0$. Computing exactly the domain of attraction is difficult. Instead, approximation of the domain of attraction is usually of use. For simplicity, in this paper, we restrict our attention to a contractive set. The definition of a contractive set for system (1) is recalled in the sequel.

Definition 4.2 Consider system (1) subject to model uncertainty (2) and constraints (3). A set $\mathcal{X} \subseteq \mathbb{X}$ is called $\lambda$-contractive for a given $0 \leq \lambda<1$ if there exists a control law $u_{k}=\kappa\left(x_{k}\right) \in \mathbb{U}$ such that

$$
\begin{aligned}
&\left(A(k) x_{k}+B(k) \kappa\left(x_{k}\right)\right) \oplus \mathbb{W} \subseteq \lambda \mathcal{X}, \\
& \forall x_{k} \in \mathcal{X}, \quad \forall[A(k) B(k)] \in \Psi .
\end{aligned}
$$

The maximal $\lambda$-contractive set, denoted as $P_{\lambda}$, is defined as the set containing all $\lambda$-contractive sets. An algorithm for the computation of the maximal $\lambda$-contractive set has been put forward in Blanchini (1994). For completeness, this algorithm is recalled below.

$$
\begin{aligned}
& S_{1}=\mathbb{X} \\
& S_{i+1}=\left\{x \in S_{i} \mid \exists u(x) \in \mathbb{U}\right. \text { s.t. } \\
& P_{\lambda}=S_{\infty} \text {. } \\
& \left.\left(A_{j} x+B_{j} u(x)\right) \oplus \mathbb{W} \subseteq \lambda S_{i}, \forall j \in \mathcal{I}_{L}\right\},
\end{aligned}
$$

Hereafter, we will use the maximal $\lambda$-contractive set as an estimation of the domain of attraction for a given $0 \leq \lambda<1$ i.e. $\mathcal{X}=P_{\lambda} \subseteq \mathbf{X}$. Without loss of generality, we assume that $\Omega \subset P_{\lambda}$.

\subsection{Convex liftings construction}

In control theory, convex liftings have been of use to facilitate implementation of piecewise affine control laws Baotic et al. (2008). Recently, they have been of use to solve the inverse parametric linear/quadratic programming problems Nguyen et al. (2014b,a, 2015b,c,d). In this paper, we will show that such convex liftings can also serve as control Lyapunov functions. Before recalling the definition of a convex lifting, additional definitions need to be recalled.

Definition 4.3 A collection of $N$ full-dimensional polyhedra $\mathcal{X}_{i} \subset \mathbb{R}^{d_{x}}$, denoted by $\left\{\mathcal{X}_{i}\right\}_{i \in \mathcal{I}_{N}}$, is called a polyhedral partition of a polyhedron $\mathcal{X} \subseteq \mathbb{R}^{d_{x}}$ if the following conditions hold:

- $\bigcup_{i \in \mathcal{I}_{N}} \mathcal{X}_{i}=\mathcal{X}$

- $\operatorname{int}\left(\mathcal{X}_{i}\right) \cap \operatorname{int}\left(\mathcal{X}_{j}\right)=\emptyset, \forall(i, j) \in \mathcal{I}_{N}^{2}, i \neq j$.

Two regions $\mathcal{X}_{i}, \mathcal{X}_{j}$ are called neighboring or adjacent if $i \neq j,(i, j) \in \mathcal{I}_{N}^{2}, \operatorname{dim}\left(\mathcal{X}_{i} \cap \mathcal{X}_{j}\right)=d_{x}-1$. Further, if $\mathcal{X}$ is a polytope, then $\left\{\mathcal{X}_{i}\right\}_{i \in \mathcal{I}_{N}}$ is called a polytopic partition.
Definition 4.4 Given a polyhedral partition $\left\{\mathcal{X}_{i}\right\}_{i \in \mathcal{I}_{N}}$ of a polyhedron $\mathcal{X} \subseteq \mathbb{R}^{d}$, a piecewise affine lifting is described by function $z: \mathcal{X} \rightarrow \mathbb{R}$ with:

$$
z(x)=a_{i}^{T} x+b_{i} \text { for any } x \in \mathcal{X}_{i},
$$

and $a_{i} \in \mathbb{R}^{d}, b_{i} \in \mathbb{R}, \forall i \in \mathcal{I}_{N}$.

Definition 4.5 Given a polyhedral partition $\left\{\mathcal{X}_{i}\right\}_{i \in \mathcal{I}_{N}}$ of a polyhedron $\mathcal{X} \subseteq \mathbb{R}^{d}$, a piecewise affine lifting $z(x)=$ $a_{i}^{T} x+b_{i}$ for $x \in \overline{\mathcal{X}}_{i}$, is called convex piecewise affine lifting if the following conditions hold true:

- $z(x)$ is continuous over $\mathcal{X}$,

- for each $i \in \mathcal{I}_{N}, z(x)>a_{j}^{T} x+b_{j}$ for all $x \in \mathcal{X}_{i} \backslash \mathcal{X}_{j}$ and all $j \neq i, j \in \mathcal{I}_{N}$.

Note that the second condition in this definition implies that any pair of neighboring regions are lifted onto two distinct hyperplanes. Also, it implies the convexity of this piecewise affine lifting. For ease of presentation, a slight abuse of notation is used hereafter: a convex lifting will be understood as a convex piecewise affine lifting.

We present now an algorithm to construct a class of convex liftings which will be of use later in the proposed robust control design. Let $\ell(x)$ denote this convex lifting defined over an estimation of the domain of attraction $\mathcal{X}$. As discussed in Subsection 4.2, we restrict our attention to the maximal $\lambda$-contractive set $P_{\lambda}$ for a given $0 \leq \lambda<1$ i.e. $\mathcal{X}=P_{\lambda}$.

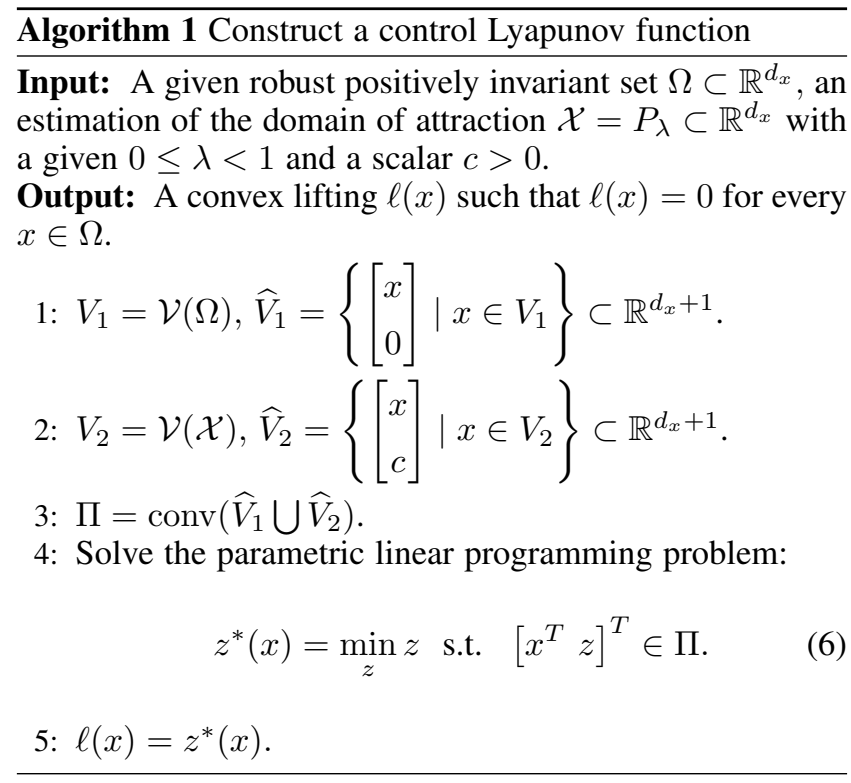

Steps 1-2 in Algorithm 1 aim to lift the vertices of $\Omega$ and $\mathcal{X}$ to $\mathbb{R}^{d_{x}+1}$ with appropriate heights. Namely, the vertices of $\Omega$ are lifted with heights equal to 0 , whereas the vertices of $\mathcal{X}$ are lifted with heights equal to the given $c>0$. Note that 
(6) is a parametric linear programming problem, its optimal solution is thus a piecewise affine function defined over a polytopic partition denoted as follows: $\ell(x)=z^{*}(x)=$ $a_{i}^{T} x+b_{i}$ for $x \in \mathcal{X}_{i}$. Note also that by construction, there exists a region in the partition associated with $\ell(x)$ which coincides with $\Omega$, since the vertices of $\Omega$ are lifted onto a lower facet of $\Pi$. The following observation describes the properties of such an $\ell(x)$, generated from Algorithm 1 .

Lemma 4.6 The function $\ell(x)$ over $\mathcal{X}$, generated from Algorithm 1, is continuous, convex, piecewise affine function.

PROOF. $\ell(x)$ is a piecewise affine function since it is induced from a parametric linear programming problem. The continuity and convexity of $\ell(x)$ can be easily derived from Theorems IV.3 and IV.4 in Gal (1995).

Lemma 4.7 The function $\ell(x)$ over $\mathcal{X}$, generated from Algorithm 1 , is a convex lifting over the associated partition $\left\{\mathcal{X}_{i}\right\}_{i \in \mathcal{I}_{N}}$.

PROOF. To prove that $\ell(x)$ is a convex lifting for $\left\{\mathcal{X}_{i}\right\}_{i \in \mathcal{I}_{N}}$, we need to prove that for any pair of $\left(\mathcal{X}_{i}, \mathcal{X}_{j}\right)$ the associated optimal solutions are different i.e. $\left(a_{i}, b_{i}\right) \neq\left(a_{j}, b_{j}\right)$. Suppose the converse situation happens, more precisely, there exist two regions $\left(\mathcal{X}_{i}, \mathcal{X}_{j}\right)$ such that $\left(a_{i}, b_{i}\right)=\left(a_{j}, b_{j}\right)$.

First, it can be easily seen that the optimal solution to the parametric linear programming problem (6) is unique. In fact, suppose there exist two different optimal solutions to (6) i.e. $z_{1}^{*}(x)$ and $z_{2}^{*}(x)$. Consider a region $\mathcal{X}_{i}$ in the associated partition over which $z_{1}^{*}(x), z_{2}^{*}(x)$ are defined i.e. $z_{1}^{*}(x)=$ $\left(a_{i}^{(1)}\right)^{T} x+b_{i}^{(1)}, z_{2}^{*}(x)=\left(a_{i}^{(2)}\right)^{T} x+b_{i}^{(2)}$. Since $z$ is the cost function of (6), therefore, we obtain:

$$
\left(a_{i}^{(1)}\right)^{T} x+b_{i}^{(1)}=\left(a_{i}^{(2)}\right)^{T} x+b_{i}^{(2)} \text { for all } x \in \mathcal{X}_{i} .
$$

Note that the set of all $x$ satisfying (7) describes a set of dimension lower than $d_{x}$, whereas (7) also holds true for all $x \in \mathcal{X}_{i}$ as a full dimensional polyhedron. This case only holds if $\left(a_{i}^{(1)}, b_{i}^{(1)}\right)=\left(a_{i}^{(2)}, b_{i}^{(2)}\right)$. This leads to the uniqueness of the optimal solution to (6).

Consider now two regions $\left(\mathcal{X}_{i}, \mathcal{X}_{j}\right)$ such that $\left(a_{i}, b_{i}\right)=$ $\left(a_{j}, b_{j}\right)$. Let the optimization problem (6) be written in the following form:

$$
\min _{z} z \text { s.t. } G z \leq W+E x .
$$

Without loss of generality, the constraint set of (8) is assumed to be in minimal representation. Also, suppose the constraints active at $\left[x^{T} a_{i}^{T} x+b_{i}\right]^{T}$ and $\left[\begin{array}{ll}x^{T} & a_{j}^{T} x+b_{j}\end{array}\right]^{T}$ are respectively as follows:

$$
\begin{aligned}
& G^{(i)} z=W^{(i)}+E^{(i)} x \\
& G^{(j)} z=W^{(j)}+E^{(j)} x .
\end{aligned}
$$

According to the uniqueness of the optimal solution to (8), $G^{(i)}, G^{(j)} \in \mathbb{R} \backslash\{0\}$. Also, since $\mathcal{X}_{i} \neq \mathcal{X}_{j}$, thus $G^{(j)} z \leq$ $W^{(j)}+E^{(j)} x$ is not active at $\left[x^{T} a_{i}^{T} x+b_{i}\right]^{T}$ for $x \in \mathcal{X}_{j}$; more precisely

$$
G^{(j)}\left(a_{i}^{T} x+b_{i}\right)<W^{(j)}+E^{(j)} x .
$$

However, as assumed $\left(a_{i}, b_{i}\right)=\left(a_{j}, b_{j}\right)$, then $G^{(j)} z \leq$ $W^{(j)}+E^{(j)} x$ becomes active at $\left[x^{T} a_{i}^{T} x+b_{i}\right]^{T}$ for $x \in$ $\mathcal{X}_{j} ;$ namely,

$$
G^{(j)}\left(a_{i}^{T} x+b_{i}\right)=W^{(j)}+E^{(j)} x .
$$

Inclusions (9) and (10) are clearly contradictory. In other words, for any pair of different regions $\left(\mathcal{X}_{i}, \mathcal{X}_{j}\right)$, the optimal solution to (6) i.e. $\ell(x)$ satisfies $\left(a_{i}, b_{i}\right) \neq\left(a_{j}, b_{j}\right)$.

Additionally, Lemma 4.6 shows that $\ell(x)$ is a continuous, convex, piecewise affine function. Therefore, $\ell(x)$ is a convex lifting for $\left\{\mathcal{X}_{i}\right\}_{i \in \mathcal{I}_{N}}$ according to Definition 4.5.

Lemma 4.8 The function $\ell(x)$ over $\mathcal{X}$, generated from Algorithm 1, satisfies $\ell(x)=0$ for every $x \in \Omega$ and $\ell(x)>0$ for all $x \in \mathcal{X} \backslash \Omega$.

PROOF. Indeed, consider $x \in \Omega$, then $x$ can be written as a convex combination of the vertices of $\Omega$ as: $x=$ $\sum_{v \in \mathcal{V}(\Omega)} \alpha(v) v$ with $\alpha(v) \geq 0$ and $\sum_{v \in \mathcal{V}(\Omega)} \alpha(v)=1$. It is known that $\ell(x)$ over $\Omega$ is an affine function, then $\ell(x)=a_{i}^{T} x+b_{i}$ leads to $\ell(x)=0$ for every $x \in \Omega$.

To complete the proof, it is necessary to show that $\ell(x)>0$ for $x \in \mathcal{X} \backslash \Omega$. Indeed, as shown above, $\ell(x)=a_{i}^{T} x+b_{i}=0$ for every $x \in \Omega$, then since $\Omega$ is full-dimensional, it follows $a_{i}=0, b_{i}=0$. Consider a region $\mathcal{X}_{j} \neq \Omega=\mathcal{X}_{i}$ of the polytopic partition $\left\{\mathcal{X}_{i}\right\}_{i \in \mathcal{I}_{N}}$ associated with $\ell(x), \ell(x)=$ $a_{j}^{T} x+b_{j}$ for every $x \in \mathcal{X}_{j}$. According to Lemma 4.7, $\ell(x)$ satisfies the convexity and continuity conditions of a convex lifting:

$$
\begin{aligned}
& a_{j}^{T} x+b_{j}>a_{i}^{T} x+b_{i}=0, \text { for every } \quad x \in \mathcal{X}_{j} \backslash \mathcal{X}_{i}, \\
& a_{j}^{T} x+b_{j}=a_{i}^{T} x+b_{i}=0, \text { for every } x \in \mathcal{X}_{j} \cap \mathcal{X}_{i}
\end{aligned}
$$

The same inclusion for the other affine functions of $\ell(x)$, leads to the non-negativity of $\ell(x)$. Moreover, $\ell(x)>0$ for every $x \in \mathcal{X} \backslash \Omega$. The proof is complete. 
Another property of $\ell(x)$ is presented as follows.

Lemma 4.9 For any $x \in \mathcal{X}$ and $0 \leq \beta \leq 1, \ell(\beta x) \leq$ $\beta \ell(x)$.

PROOF. Due to the convexity of $\ell(x)$ over $\mathcal{X}$ as proved in Lemma 4.6, it leads to

$$
\ell(\beta x+(1-\beta) 0) \leq \beta \ell(x)+(1-\beta) \ell(0) .
$$

Due to the assumption that $0 \in \operatorname{int}(\mathbb{W})$, then $0 \in \operatorname{int}(\Omega)$, meaning that $\ell(0)=0$ according to Lemma 4.8. This inclusion and the above one imply that $\ell(\beta x) \leq \beta \ell(x)$.

\subsection{Robust control design procedure}

This subsection introduces the procedure for designing robust control laws based on convex liftings. This procedure can guarantee robust stability of the closed loop in the sense of Lyapunov. Therefore, a definition of this robust stability is recalled below.

Definition 4.10 Given a robust positively invariant set $\Omega$ and the domain of attraction $\mathcal{X} \subseteq \mathbb{X}$, consider the linear system (1) subject to constraints (3) and a control law $u=$ $\kappa(x) \in \mathbb{U}$. The closed loop is called robustly stable if there exists a Lyapunov function $V(x): \mathcal{X} \rightarrow \mathbb{R}_{+}$and an $\alpha \in$ $[0,1)$ such that:

- $V(x)=0$ for all $x \in \Omega, V(x)>0$ for all $x \in \mathcal{X} \backslash \Omega$,

- $V\left(A(k) x_{k}+B(k) \kappa\left(x_{k}\right)+w_{k}\right)-\alpha V\left(x_{k}\right) \leq 0, \forall w_{k} \in \mathbb{W}$, $\forall x_{k} \in \mathcal{X} \backslash \Omega$ and $\forall[A(k) B(k)] \in \Psi$.

For robust design based on control Lyapunov function, it is important to find such a control Lyapunov function and use it for design procedure. Our design procedure based on a convex lifting, computed from Algorithm 1, is summarized in Algorithm 2.

Remark 4.11 Note that the task of verifying whether or not $x_{k}$ belongs to $\Omega$ in Step 2 of Algorithm 2, can be easily carried out by checking whether or not $\ell\left(x_{k}\right)=0$. This property is due to the construction of a convex lifting from Algorithm 1. Therefore, it is not necessary to store the constraints describing $\Omega$ in the implementation.

Natural questions arise here whether or not the linear programming problem (11) is feasible and whether closed loop stability is guaranteed by the proposed procedure. These questions are answered via the following theorem. Accordingly, it will be shown that convex lifting constructed in Algorithm 1 can serve as a Lyapunov function. Thus, the proposed control design can guarantee the robust stability as per Definition 4.10. $\overline{\text { Algorithm } 2 \text { Robust control design procedure based on con- }}$ vex liftings

Input: A robust positively invariant set $\Omega$ associated with a stabilizing control law $u=K x$ over $\Omega$. A convex lifting $\ell(x)=a_{i}^{T} x+b_{i}$ for $x \in \mathcal{X}_{i}, i \in \mathcal{I}_{N}$ as in Algorithm 1 .

Output: Control law $u^{*}\left(x_{k}\right)$ at each sampling time.

1: Compute $\ell\left(x_{k}\right)$.

2: If $x_{k} \in \Omega$ then $u^{*}\left(x_{k}\right)=K x_{k}$, jump to Step 6 .

3: Else Solve the following linear programming problem:

$$
\begin{array}{cl} 
& {\left[\alpha^{*}\left(u_{k}^{*}\right)^{T}\right]^{T}=\arg \min _{\alpha, u_{k}} \alpha} \\
\text { s.t. } & a_{i}^{T}\left(A_{j} x_{k}+B_{j} u_{k}+w\right)+b_{i} \leq \alpha \ell\left(x_{k}\right) \\
& \alpha \geq 0, u_{k} \in \mathbb{U}, \forall i \in \mathcal{I}_{N}, \forall w \in \mathcal{V}(\mathbb{W}), \\
& \forall\left[A_{j} B_{j}\right] \in \mathcal{V}(\Psi) .
\end{array}
$$

4: Apply $u^{*}\left(x_{k}\right)=u_{k}^{*}$

5: End

6: $k \leftarrow k+1$, return to Step 1 .

Theorem 4.12 Given a robust positively invariant set $\Omega$ associated with a robust control law gain $K$ and an estimation of the domain of attraction $\mathcal{X}=P_{\lambda}$ for a given $0 \leq \lambda<1$, if the initial condition $x_{k} \in \mathcal{X}$, then the linear programming problem (11) is recursively feasible. Furthermore, the closed loop is robustly stable in the sense of Lyapunov.

PROOF. As for the feasibility of (11), one can easily see that $0 \leq \ell(x) \leq c$ by the construction in Algorithm 1 . Therefore, due to the contractivity of $\mathcal{X}$, for any $x_{k} \in \mathcal{X}$ there always exists $u\left(x_{k}\right) \in \mathbb{U}$ such that:

$$
A(k) x_{k}+B(k) u\left(x_{k}\right)+w_{k} \in \lambda \mathcal{X} \subset \mathcal{X}
$$

for all $w_{k} \in \mathbb{W}$ and for all $[A(k) B(k)] \in \Psi$. Therefore, if $u^{*}\left(x_{k}\right)$ denotes an optimal solution to (11), then one has:

$$
\begin{aligned}
0 & \leq \ell\left(A(k) x_{k}+B(k) u^{*}\left(x_{k}\right)+w_{k}\right) \\
& \leq \ell\left(A(k) x_{k}+B(k) u\left(x_{k}\right)+w_{k}\right) \\
& \leq c, \quad \forall w_{k} \in \mathbb{W}, \quad \forall[A(k) B(k)] \in \Psi .
\end{aligned}
$$

Due to this boundedness, the recursive feasibility of the linear programming problem (11) is ensured for a finite, large enough scalar $\alpha$ at each sampling time.

As for robust stability, it will be proved that for all $x_{k} \in$ $\mathcal{X} \backslash \Omega$ :

$$
\begin{gathered}
\ell\left(A(k) x_{k}+B(k) u^{*}\left(x_{k}\right)+w_{k}\right)<\ell\left(x_{k}\right), \\
\forall w_{k} \in \mathbb{W}, \forall[A(k) B(k)] \in \Psi .
\end{gathered}
$$

Indeed, due to the contractivity of $\mathcal{X}$, for any $v \in \mathcal{V}(\mathcal{X})$, there exists a control law, denoted by $u(v) \in \mathbb{U}$ such that $A(k) v+B(k) u(v)+w_{k} \in \lambda \mathcal{X}$ despite any disturbances $w_{k} \in \mathbb{W}$ and for all $[A(k) B(k)] \in \Psi$. For each $w_{k} \in \mathbb{W}$ 
and each $[A(k) B(k)] \in \Psi$, there exists $y\left(k, w_{k}\right) \in \mathcal{X}$ such that

$$
A(k) v+B(k) u(v)+w_{k}=\lambda y\left(k, w_{k}\right) .
$$

Due to Lemma 4.9, this inclusion leads to

$$
\begin{aligned}
\ell\left(A(k) v+B(k) u(v)+w_{k}\right) & =\ell\left(\lambda y\left(k, w_{k}\right)\right) \\
& \leq \lambda \ell\left(y\left(k, w_{k}\right)\right) .
\end{aligned}
$$

By the construction of $\ell(x)$ in Algorithm 1, the following is obtained:

$$
\ell\left(y\left(k, w_{k}\right)\right) \leq c .
$$

Also, according to Algorithm 1,

$$
\ell(v)=c .
$$

From (12), (13), (14), one can deduce that

$$
\ell\left(A(k) v+B(k) u(v)+w_{k}\right) \leq \lambda \ell(v)
$$

Note that (15) holds for all $w_{k} \in \mathbb{W}$ and for all $[A(k) B(k)] \in \Psi$. Moreover, it can be observed that:

$\ell\left(A(k) v+B(k) u^{*}(v)+w_{k}\right) \leq \ell\left(A(k) v+B(k) u(v)+w_{k}\right)$, $\forall w_{k} \in \mathbb{W}, \forall[A(k) B(k)] \in \Psi$,

where $u^{*}(x)$ denotes optimal control to (11) at $x$ as used in Algorithm 2. (15) and (16) lead to the following fact:

$$
\begin{array}{r}
\ell\left(A(k) v+B(k) u^{*}(v)+w_{k}\right) \leq \lambda \ell(v), \\
\forall w_{k} \in \mathbb{W}, \forall[A(k) B(k)] \in \Psi .
\end{array}
$$

Note that (17) holds true for all vertices of $\mathcal{X}$. Now, consider a point $x_{k} \in \mathcal{X}_{i}$ in the polytopic partition $\left\{\mathcal{X}_{i}\right\}_{i \in \mathcal{I}_{N}}$ of $\mathcal{X}$ over which $\ell(x)$ is defined. Without loss of generality, suppose $\mathcal{X}_{i} \neq \Omega$, then $x_{k}$ can be described via a convex combination of the vertices of $\mathcal{X}_{i}$, meaning:

$x_{k}=\sum_{v \in \mathcal{V}\left(\mathcal{X}_{i}\right)} \alpha(v) v$, where $\alpha(v) \in \mathbb{R}_{+}, \sum_{v \in \mathcal{V}\left(\mathcal{X}_{i}\right)} \alpha(v)=1$.

Recall that due to the definition of convex lifting, $\ell(x)$ over $\mathcal{X}_{i}$ is an affine function, then $\ell\left(x_{k}\right)$ can be written in the following form:

$$
\ell\left(x_{k}\right)=\sum_{v \in \mathcal{V}\left(\mathcal{X}_{i}\right)} \alpha(v) \ell(v)
$$

If $v \in \mathcal{V}\left(\mathcal{X}_{i}\right)$ is a vertex of $\Omega$, then due to the robust positive invariance of $\Omega$ with respect to a linear feedback $u^{*}(x)=$ $K x$, it satisfies

$$
\begin{array}{r}
\ell(v)=0=\ell\left((A(k)+B(k) K) v+w_{k}\right), \\
\forall w_{k} \in \mathbb{W}, \forall[A(k) B(k)] \in \Psi .
\end{array}
$$

Otherwise, if $v \in \mathcal{V}\left(\mathcal{X}_{i}\right)$ is a vertex of $\mathcal{X}$, then it satisfies (17). Therefore, due to the convexity of $\ell(x)$ proved in Lemma 4.6 and (17), (18), (19), the following is obtained:

$$
\begin{aligned}
& \lambda \ell\left(x_{k}\right)=\sum_{v \in \mathcal{V}\left(\mathcal{X}_{i}\right)} \alpha(v)(\lambda \ell(v)) \\
& \geq \sum_{v \in \mathcal{V}\left(\mathcal{X}_{i}\right)} \alpha(v) \ell\left(A(k) v+B(k) u^{*}(v)+w_{k}\right) \\
& \geq \ell\left(A(k) \sum_{v \in \mathcal{V}\left(\mathcal{X}_{i}\right)} \alpha(v) v+B(k) \sum_{v \in \mathcal{V}\left(\mathcal{X}_{i}\right)} \alpha(v) u^{*}(v)+w_{k}\right) \\
& =\ell\left(A(k) x_{k}+B(k) \sum_{v \in \mathcal{V}\left(\mathcal{X}_{i}\right)} \alpha(v) u^{*}(v)+w_{k}\right) .
\end{aligned}
$$

Recall that $u^{*}(v) \in \mathbb{U}, \forall v \in \mathcal{V}\left(\mathcal{X}_{i}\right) \cap \mathcal{V}(\mathcal{X})$ and $u^{*}(v)=$ $K v \in \mathbb{U}, \forall v \in \mathcal{V}\left(\mathcal{X}_{i}\right) \cap \mathcal{V}(\Omega)$, then it follows that

$$
\sum_{v \in \mathcal{V}\left(\mathcal{X}_{i}\right)} \alpha(v) u^{*}(v) \in \mathbb{U}
$$

Therefore, (21) leads to:

$$
\begin{aligned}
\ell\left(A(k) x_{k}+B(k) \sum_{v \in \mathcal{V}\left(\mathcal{X}_{i}\right)} \alpha(v) u^{*}(v)+w_{k}\right) \\
\geq \ell\left(A(k) x_{k}+B(k) u^{*}\left(x_{k}\right)+w_{k}\right) .
\end{aligned}
$$

From (20) and (22), the following inclusion can be obtained:

$$
\begin{array}{r}
\lambda \ell\left(x_{k}\right) \geq \ell\left(A(k) x_{k}+B(k) u^{*}\left(x_{k}\right)+w_{k}\right), \\
\forall w_{k} \in \mathbb{W}, \forall[A(k) B(k)] \in \Psi .
\end{array}
$$

Recall that $0 \leq \lambda<1$, therefore

$$
\begin{array}{r}
\ell\left(x_{k}\right)>\ell\left(A(k) x_{k}+B(k) u^{*}\left(x_{k}\right)+w_{k}\right), \\
\forall w_{k} \in \mathbb{W}, \forall[A(k) B(k)] \in \Psi,
\end{array}
$$

meaning $\left\{\ell\left(x_{k}\right)\right\}_{k=0}^{\infty}$ is a strictly decreasing sequence outside $\Omega$ and bounded in the interval $[0, c]$. Thus, this sequence is convergent to 0 . In other words, $\ell(x)$ serves as a Lyapunov function according to Definition 4.10.

Remark 4.13 Note that by construction, the partition associated with a convex lifting in Algorithm 1, may not be a Delaunay decomposition as in Scibilia et al. (2009). This method does not rely on such a decomposition, but relies on a convex lifting defined over this partition. This approach is simple and only requires solving a linear programming problem at each sampling instant. However, the associated control law is not continuous at the moment the state switches into $\Omega$ (see step 2 of Algorithm 2). Note also that the checking whether the current state belongs to $\Omega$ can be relaxed. Accordingly, one can continue solving the problem (11) while trajectories still stay inside $\Omega$. Indeed, if $x_{k} \in \Omega$, then due 
to the construction $\ell\left(x_{k}\right)=0$. Consider the next state, one can see that $K x_{k} \in \mathbb{U}$, then it leads to:

$$
\begin{aligned}
0 & \leq \ell\left(A(k) x_{k}+B(k) u^{*}\left(x_{k}\right)+w_{k}\right) \\
& \leq \ell\left(A(k) x_{k}+B(k) K x_{k}+w_{k}\right)=0=\ell\left(x_{k}\right) .
\end{aligned}
$$

This inclusion implies that optimal control law $u^{*}\left(x_{k}\right) \in \mathbb{U}$ to problem (11) also keeps the trajectories inside $\Omega$, if $x_{k}$ is inside $\Omega$.

Remark 4.14 An open problem is to guarantee robust stability of the proposed method for another estimation of the domain of attraction as the $N$-steps robust controllable set denoted by $\mathcal{K}_{N}(\Omega)$ c.f. Kerrigan (2001). Note that in this case, proving the strict decrease of $\ell(x)$ becomes more difficult. Also, this strict decrease may not be successive.

Remark 4.15 Note that the explicit robust controller of (11) can be obtained by replacing $\alpha \ell\left(x_{k}\right)$ with a variable, denoted by e.g. $z$. Accordingly, the optimization problem (11) becomes a parametric linear programming problem with the decision argument to be $\left[z u_{k}^{T}\right]^{T}$ and the parameter as the current state $x_{k}$.

\section{Numerical examples}

To illustrate the proposed procedure, consider the following uncertain system:

$$
x_{k+1}=A(k) x_{k}+B(k) u_{k},
$$

where

$$
\begin{aligned}
& {[A(k) B(k)] \in \operatorname{conv}\left\{\left[\begin{array}{ccc}
1 & 0.1 & 0 \\
0 & 0.9 & 0.1
\end{array}\right],\left[\begin{array}{ccc}
1 & 0.1 & 0 \\
0 & 0 & 0.1
\end{array}\right]\right\},} \\
& {[A(k) B(k)]=\beta_{k}\left[\begin{array}{ccc}
1 & 0.1 & 0 \\
0 & 0.9 & 0.1
\end{array}\right]+\gamma_{k}\left[\begin{array}{ccc}
1 & 0.1 & 0 \\
0 & 0 & 0.1
\end{array}\right],}
\end{aligned}
$$

and $\beta_{k}=\sin ^{2}\left(p_{k}\right), \gamma_{k}=\cos ^{2}\left(p_{k}\right), p_{k}$ represents a random scalar variable at time $k$. The present state and control variables are subject to the following constraints:

$$
\left[\begin{array}{l}
-10 \\
-10
\end{array}\right] \leq x_{k} \leq\left[\begin{array}{l}
10 \\
10
\end{array}\right],-5 \leq u_{k} \leq 5
$$

An unconstrained controller is chosen as follows:

$$
u=[-3.2827-4.6780] x .
$$

Accordingly, the maximal robust positively invariant set associated with the above controller, i.e. $\Omega$ is shown in Fig. 1.
Also, the maximal 0.99 -contractive set $P_{0.99}$ is presented therein. This set is computed from procedure (4). A convex lifting $\ell(x)$, serving later as a control Lyapunov function, is visualized in Fig. 2 according to Algorithm 1 with $c=10$. The closed loop trajectories are shown in Fig. 3 to be convergent to the origin, since the unconstrained control law can cope with the given set of polytopic uncertainties over $\Omega$. Finally, the strict decrease of $\ell\left(x_{k}\right)$ over $\mathcal{X} \backslash \Omega$, is illustrated in Fig. 4. The numerical example of this paper has

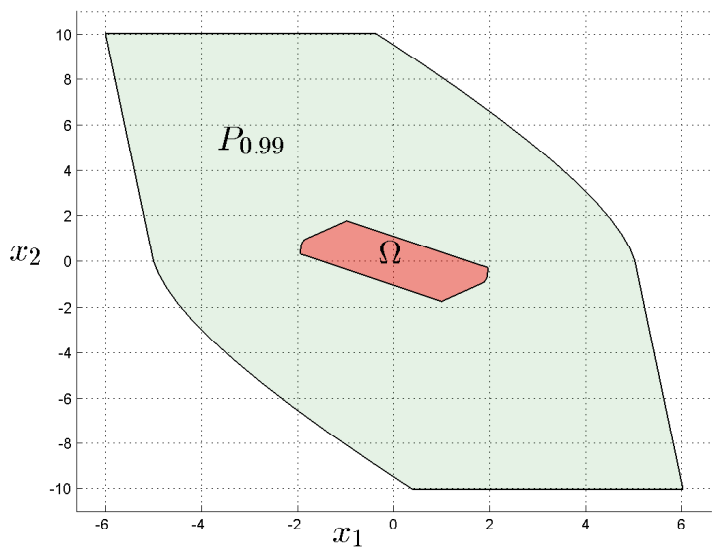

Fig. 1. The maximal robust positively invariant set $\Omega$ and an estimation of the domain of attraction $\mathcal{X}=P_{0.99}$.

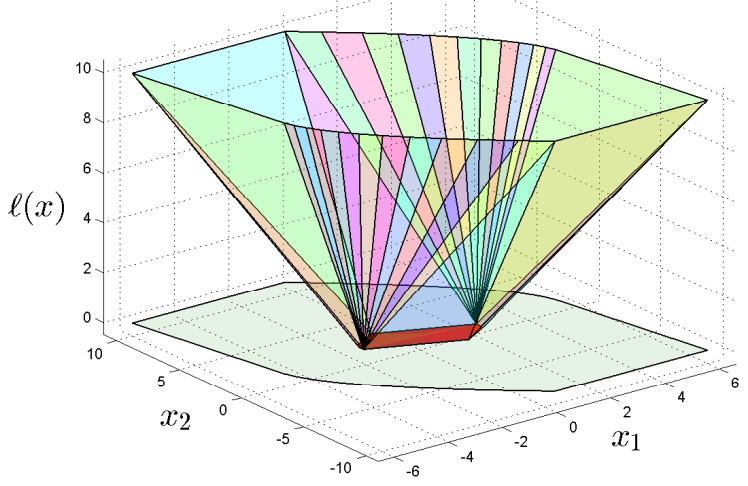

Fig. 2. A convex lifting $\ell(x)$ constructed by Algorithm 1 with $c=10$.

been simulated in the environment of MPT 3.0 Herceg et al. (2013).

\section{Conclusions}

This paper presented a new method to design robust control law for constrained linear systems affected by bounded additive disturbances and polytopic uncertainties. This method was based on convex liftings. It was shown to guarantee the 


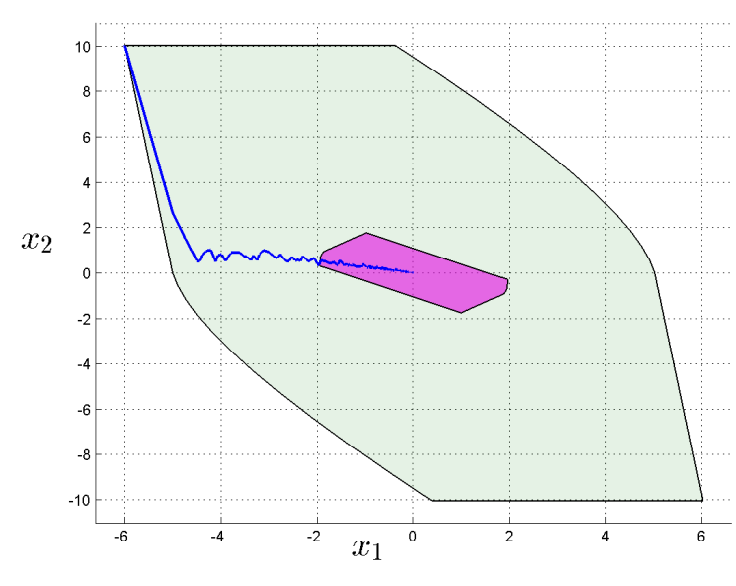

Fig. 3. Closed loop stability.

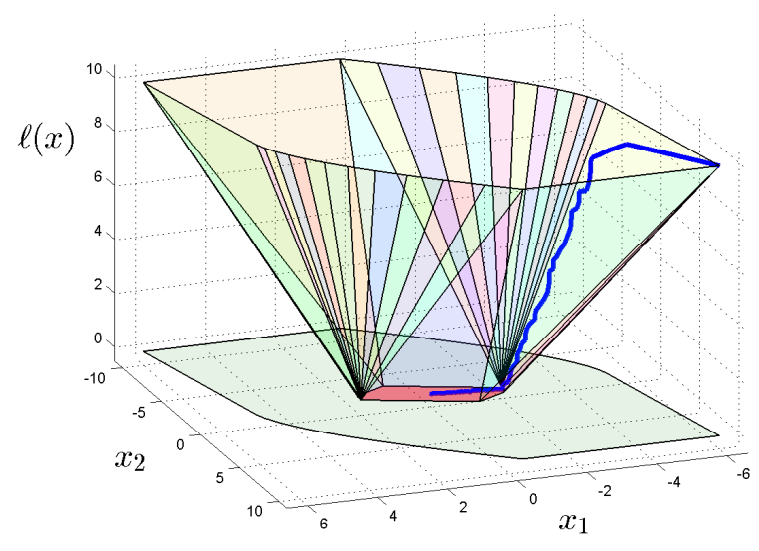

Fig. 4. The strict decrease of $\ell(x)$ over $\mathcal{X} \backslash \Omega$ along the state.

recursive feasibility and also robust stability in the sense of Lyapunov.

\section{References}

Anderson, B.D., Moore, J.B., 2007. Optimal control: linear quadratic methods. Courier Corporation.

Baotic, M., Borrelli, F., Bemporad, A., Morari, M., 2008. Efficient on-line computation of constrained optimal control. SIAM Journal on Control and Optimization 47, 2470-2489.

Bitsoris, G., 1988a. On the positive invariance of polyhedral sets for discrete-time systems. Systems \& control letters 11, 243-248.

Bitsoris, G., 1988b. Positively invariant polyhedral sets of discrete-time linear systems. International Journal of Control 47, 1713-1726.

Bitsoris, G., Vassilaki, M., 1995. Constrained regulation of linear systems. Automatica 31, 223-227.

Blanchini, F., 1994. Ultimate boundedness control for uncertain discrete-time systems via set-induced lyapunov functions. Automatic Control, IEEE Transactions on 39, 428-433.

Blanchini, F., 1995. Nonquadratic lyapunov functions for robust control. Automatica 31, 451-461.

Blanchini, F., Miani, S., 2007. Set-theoretic methods in control. Springer.

Boyd, S.P., El Ghaoui, L., Feron, E., Balakrishnan, ., 1994. Linear matrix inequalities in system and control theory. volume 15. SIAM.

Chmielewski, D., Manousiouthakis, V., 1996. On constrained infinite-time linear quadratic optimal control. Systems \& Control Letters 29, 121 - 129.

Gal, T., 1995. Postoptimal analyses, parametric programming and related topics. Walter de Gruyter.

Gilbert, E.G., Tan, K.T., 1991. Linear systems with state and control constraints: The theory and application of maximal output admissible sets. Automatic Control, IEEE Transactions on 36, 1008-1020.

Grammatico, S., Blanchini, F., Caiti, A., 2014. Controlsharing and merging control lyapunov functions. Automatic Control, IEEE Transactions on 59, 107-119.

Gutman, P.O., Cwikel, M., 1987. An algorithm to find maximal state constraint sets for discrete-time linear dynamical systems with bounded controls and states. Automatic Control, IEEE Transactions on 32, 251-254.

Herceg, M., Kvasnica, M., Jones, C., Morari, M., 2013. Multi-parametric toolbox 3.0, in: Control Conference (ECC), 2013 European, pp. 502-510.

Jiang, Z.P., Wang, Y., 2001. Input-to-state stability for discrete-time nonlinear systems. Automatica 37, 857869.

Kerrigan, E.C., 2001. Robust constraint satisfaction: Invariant sets and predictive control. Ph.D. thesis. University of Cambridge.

Khalil, H.K., 2002. Nonlinear systems. Prentice Hall, Inc , 323.

Kofman, E., Haimovich, H., Seron, M.M., 2007. A systematic method to obtain ultimate bounds for perturbed systems. International Journal of Control 80, 167-178.

Kolmanovsky, I., Gilbert, E.G., 1998. Theory and computation of disturbance invariant sets for discrete-time linear systems. Mathematical Problems in Engineering 4, 317 367.

Kothare, M.V., Balakrishnan, V., Morari, M., 1996. Robust constrained model predictive control using linear matrix inequalities. Automatica 32, 1361-1379.

Lazar, M., 2010. On infinity norms as lyapunov functions: Alternative necessary and sufficient conditions, in: Decision and Control (CDC), 2010 49th IEEE Conference on, IEEE. pp. 5936-5942.

Lyapunov, A.M., 1907. Problème général de la stabilité du mouvement, in: Annales de la Faculté des Sciences de Toulouse, Université Paul Sabatier. pp. 203-474.

Molchanov, A.P., Pyatnitskiy, Y.S., 1989. Criteria of asymptotic stability of differential and difference inclusions encountered in control theory. Systems \& Control Letters $13,59-64$.

Nguyen, H.N., 2014. Constrained Control of Uncertain, Time-Varying, Discrete-Time Systems: An Interpolation- 
Based Approach. Lecture Notes in Control and Information Sciences.

Nguyen, N.A., Olaru, S., Rodriguez-Ayerbe, 2015a. Robust control design based on convex liftings, in: the 8th IFAC Symposium on Robust Control Design.

Nguyen, N.A., Olaru, S., Rodriguez-Ayerbe, P., 2015b. Any discontinuous pwa function is optimal solution to a parametric linear programming problem, in: 54th IEEE Conference on Decision and Control, Osaka, Japan.

Nguyen, N.A., Olaru, S., Rodriguez-Ayerbe, P., 2015c. Inverse parametric linear/quadratic programming problem for continuous pwa functions defined on polyhedral partitions of polyhedra, in: 54th IEEE Conference on Decision and Control, Osaka, Japan.

Nguyen, N.A., Olaru, S., Rodriguez-Ayerbe, P., Hovd, M., Necoara, I., 2014a. Inverse parametric convex programming problems via convex liftings, in: Proc. of the 19th IFAC World Congress, Cape Town, SA.

Nguyen, N.A., Olaru, S., Rodriguez-Ayerbe, P., Hovd, M., Necoara, I., 2014b. On the lifting problems and their connections with piecewise affine control law design, in: Control Conference (ECC), 2014 European, IEEE. pp. 2164-2169.

Nguyen, N.A., Olaru, S., Rodriguez-Ayerbe, P., Hovd, M., Necoara, I., 2015d. Constructive solution of inverse parametric linear/quadratic programming problems. https://hal-centralesupelec.archives-ouvertes.fr/hal01207234/document .

Polanski, A., 1995. On infinity norms as lyapunov functions for linear systems. IEEE Transactions on Automatic Control 40, 1270-1274.

Rakovic, S.V., Kerrigan, E.C., Kouramas, K.I., Mayne, D.Q., 2005. Invariant approximations of the minimal robust positively invariant set. Automatic Control, IEEE Transactions on 50, 406-410.

Scibilia, F., Olaru, S., Hovd, M., 2009. Approximate explicit linear MPC via Delaunay tessellation, in: European Control Conference, Budapest, Hungary.

Zubov, V.I., Boron, L.F., 1964. Methods of AM Lyapunov and their application. Noordhoff Groningen. 